\title{
Ex vivo Tissue Discrimination by Visible and Near-Infrared Spectra with Chemometrics
}

\author{
Akikazu SAKUDO ${ }^{1,2)}$, Roumiana TSENKOVA ${ }^{3)}$, Kyoko TEI ${ }^{3)}$, Hiroyuki MORITA ${ }^{3)}$, Kazuyoshi IKUTA ${ }^{2)}$ and Takashi \\ ONODERA $^{1) *}$ \\ ${ }^{1)}$ Department of Molecular Immunology, School of Agricultural and Life Sciences, The University of Tokyo, Bunkyo-ku, Tokyo 113-8657, \\ ${ }^{2)}$ Department of Virology, Research Institute for Microbial Diseases, Osaka University, Yamadaoka, Suita, Osaka $565-0871$ and \\ ${ }^{3)}$ Faculty of Agriculture, Kobe University, Rokkodai, Nada-ku, Kobe 657-8501, Japan
}

(Received 19 July 2006/Accepted 29 August 2006)

ABSTRACT. The risk of infections from zoonotic pathogens of tissues and/or tissue-derived products has been increasing. One preventive approach in reducing infection risk is tissue decontamination, where selection and screening of highly infectious tissues are strictly followed. Therefore, the development of reliable analytical methods for rapid tissue discrimination is essentially important. In the present study, a procedure has been developed for intact tissue discrimination on the basis of multivariate analysis of visible and near-infrared (Vis-NIR) spectra of certain tissues such as brain, liver, kidney and testis of mice without any pretreatment. Transmittance spectra in the 600- to 1000-nm regions were subjected to a principal component analysis (PCA), and leave-out cross-validation was employed to develop multivariate models for tissue discrimination. The plot of PCA scores against Vis-NIR spectra of brains, kidneys, livers and testes from 11 mice portrayed reliable tissue discrimination. This result suggests that Vis-NIR spectroscopy combined with chemometrics analysis may provide a potentially useful approach for rapid non-destructive discrimination of tissues. KEY WORDS: principal component analysis, tissue, Vis-NIR spectroscopy.

J. Vet. Med. Sci. 68(12): 1375-1378, 2006

The use of human and animal organs, tissues and/or their derived products has been increasing in the field of medicine, agriculture and food industries. Organs and tissues such as the lung, heart, kidney, liver, cornea, cardiac values, bone, tendon, skin and cartilage are often used for transplantation [4]. In the production process of pharmaceutical products, organs and tissues are often also used. The key problem of medical, pharmaceutical and agricultural applications of organs and tissues derived from human and animals is the risk of contamination with infectious agents [5]. Furthermore, an increase in the emergence of zoonotic pathogens (i.e. animal-to-human transmission) has recently been reported [25]. Therefore, attention of the transmission risk of zoonotic pathogens has been focused on both humanand animal-derived materials. Prions (proteineous infectious particles), which cause prion diseases such as Creutzfeldt-Jakob disease (CJD) and Gerstmann-Sträussler Scheinker syndrome in humans, scrapie in sheep and goats and bovine spongiform encephalopathy (BSE), are representative of such zoonotic pathogens [17]. Infections of lyssa virus by transplants in the USA and Germany have recently been documented as well [23]. There are two major approaches to address these issues: (i) tissue decontamination with strict selection and screening of materials and donors; and (ii) inactivation and elimination of infectious pathogens to reduce transmission risks. To date, approach (i) has been constructively employed in reducing transmission risks of BSE and CJD [16]; viz., introduction of a decontamination process eliminates highly infectious patho-

\footnotetext{
* Correspondence to: Onodera, T., School of Agricultural and Life Sciences, University of Tokyo, Bunkyo-ku, Tokyo 1138657, Japan.
}

gens from tissues (e.g. brain, spinal cord or retina) and medicinal products derived from these tissues (i.e. human growth hormone, dura mater). However, inactivating methods with an autoclave, exposure to strong alkalis and/or bleaching are impractical for elimination of prions in medicinal products and foods, as their beneficial effects are susceptibly degraded by such a treatment process [16]. Furthermore, current methods [such as enzyme-linked immunosorbent assay (ELISA), Western blotting, and immunohistochemical analysis] in detecting prion diseases are time-consuming [6]. Therefore, a reliable and rapid method of discriminatively selecting donor materials, such as organs and tissues, is considered an efficient measure to reduce the transmission risk of zoonotic pathogens [7]. In short, development of analytical methods for discriminating unfit organs and tissues is essentially important in reducing risks of pathogen contamination, especially in terms of public health.

Various imaging methods, such as radiography [14], ultrasonic echo [8], magnetic resonance [3], computed tomography [11], endoscopic [11], laparoscopic [11] and intra-operative sonography [11], have been beneficially exploited as noninvasive methods for obtaining organ/tissue images; however the application of these methods is limited as they cannot be expanded for the discrimination of samples. Accordingly, higher resolution images at the cellular level are required for discrimination of tissue samples. For such purposes, histology is used. Histological analysis, however, is a destructive technique that allows one-time analysis of samples. Although useful and yet limited in their applications, the above methods each yields unique information with reference to the aspect of tissue analysis. 
Visible and near-infrared (Vis-NIR) spectroscopy is extensively employed in the agricultural, pharmaceutical, chemical, petrochemical and medical fields as a rapid noninvasive analytical method without sample preparations or use of chemical reagents $[2,18-20,22]$. The NIR and red regions are dominated by weak overtones and a combination of vibration bands of atoms with strong molecular bonds containing nitrogen, oxygen and/or carbon attached to the hydrogen atom; i.e., constituent elements of important biomolecules such as proteins, lipids and sugars [13]. The present study investigated whether the non-destructive measurement of Vis-NIR spectra combined with multivariate analysis would realize tissue discrimination.

Eleven male C57BL/6CrSlc mice (Japan SLC, Hamamatsu, Japan) were analyzed at 10 weeks of age. All mice were housed according to standard animal care protocols and sacrificed according to guidelines of the University of Tokyo before harvesting the tissues. Harvested tissues were stored in a freezer at $-80^{\circ} \mathrm{C}$ before spectroscopic measurements. The frozen tissue samples were thawed and left standing at $25^{\circ} \mathrm{C}$ to establish a stable temperature. Transmittance spectra of the tissue samples in a polystyrene cuvette (optical length: $10 \mathrm{~mm}$; SARSTEDT, Numbrecht, Germany) in a dark box were measured at 1-nm resolution with a Fruits-Tester-20 spectrophotometer (Japan Fantec Research Institute, Shizuoka, Japan) at $25^{\circ} \mathrm{C}$ (Fig. 1). Spectral data were collected to yield the absorbance value [log $(1 / T)]$, where $T$ is transmittance measured within the wavelength range of 600-1000 $\mathrm{nm}$. Three consecutive spectra were taken from each sample. Pirouette software (ver. 3.11; Infometrics, Woodinville, WA) was employed for data-processing of the Vis-NIR spectra. To minimize differences between the spectra caused by baseline shifts and noise prior to calibration, spectral data were mean-centered and transformed by smoothing before establishing the second-derivative based on the Savitsky-Golay algorithm [21, 24] and standard normal variate (SNV) [1]. The mathematical formulas are available in the Pirouette manual. The principal component analysis (PCA) was applied to develop the PCA model for assessing fundamental differences between the spectra $[9,10,12,27]$ before the PCA scores and loadings were calculated. Calculations were performed by leave-out cross-validation using 3 spectra after training other samples.

The respective transmittance Vis-NIR spectra of each organ/tissue (i.e. brains, liver, kidney, testis) of 11 mice were collected within the wavelength range of 600- to 1000$\mathrm{nm}$. To determine predominant changes in the spectra, four pre-processings were performed; viz., mean-centering, smoothing, second-derivative and SNV. Mean-centering and second-derivative emphasized the subtle variations in the spectra, while smoothing and SNV were performed to minimize noise variations. Following the determination of predominant species in the spectra, PCA of pre-processed data was performed. PCA performed to extract a quick overview of spectra reveals clusters and trends. Cross-validation was then applied to construct the PCA models. The score plots and loadings of the PCA model (Fig. 2) indicated clear discriminations of the PCA score plots [first principal component (PC1) versus third principal component (PC3) (Fig. 2A), and second principal component (PC2) versus PC3 (Fig. 2B)] in the brain, liver, kidney and testis. The spectral information modeled by the first three PCs can be inferred from the corresponding loadings. Loadings and spectra have the same dimension, and loadings may be
A

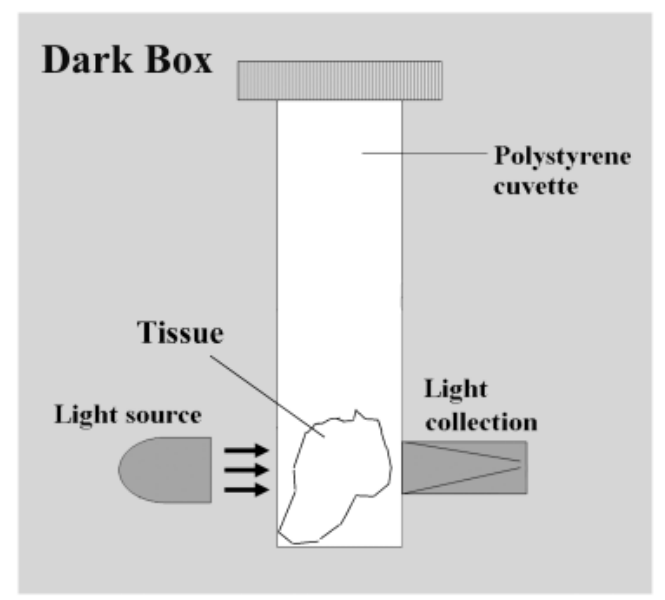

B

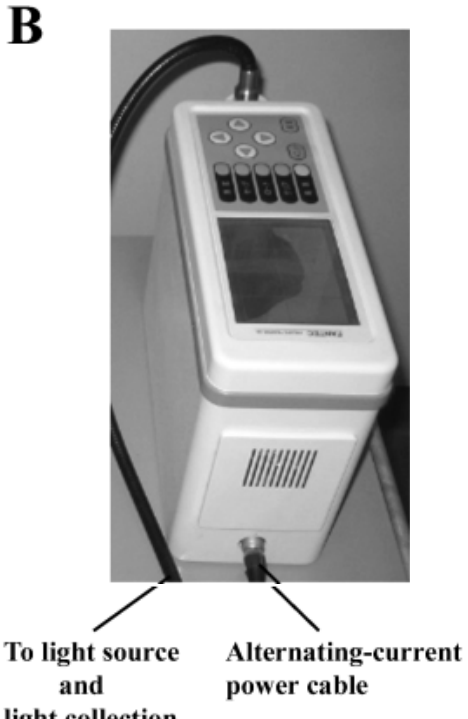

light collection

Fig. 1. Schematic representation of non-destructive measurement of a tissue sample by transmittance mode. Three consecutive transmittance of visible and near-infrared (Vis-NIR) spectra of each tissue in a polystyrene cuvette placed in a dark box (A) were collected by a Fruits-Tester-20 spectrophotometer (B) equipped with light fiber optics in an air-conditioned room at $25^{\circ} \mathrm{C}$. The light source and light collector were attached to the end of light fiber optics. 

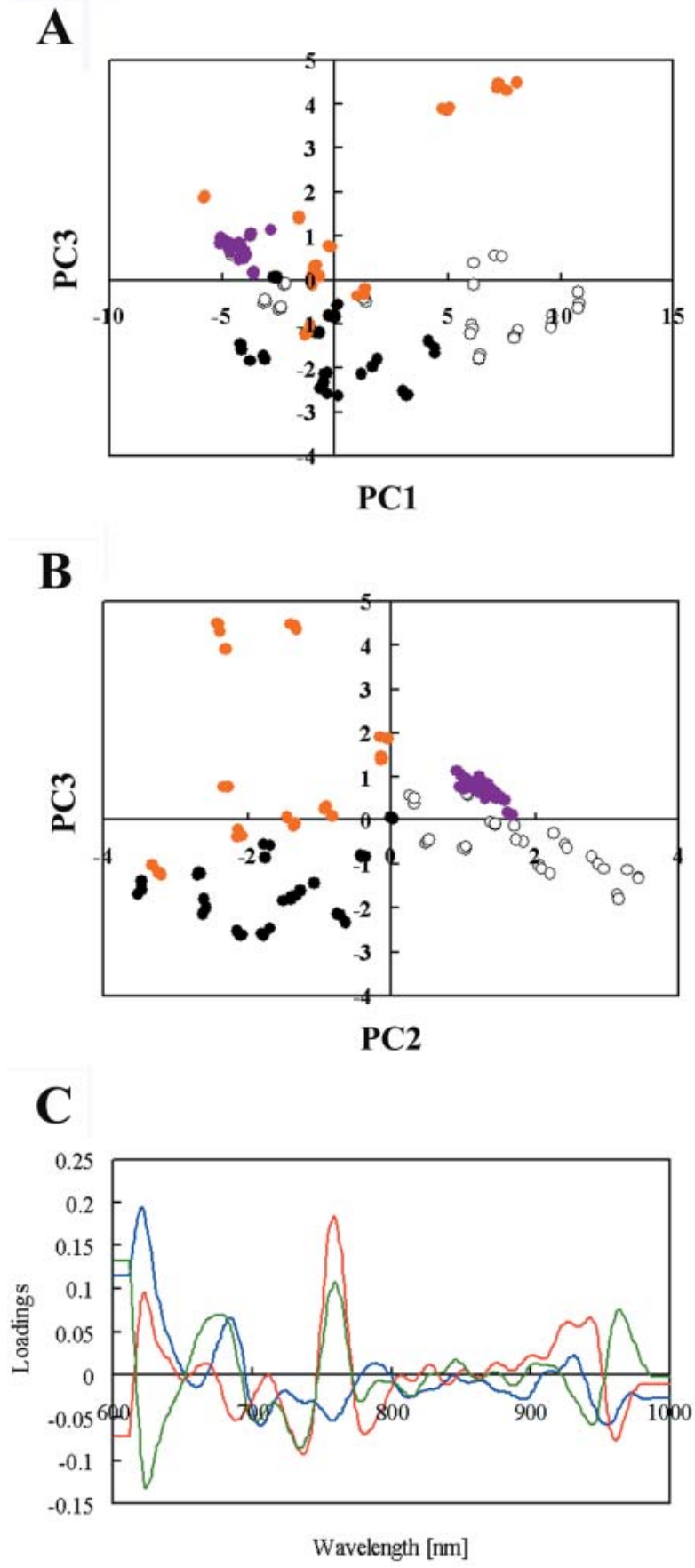

Fig. 2. Principal component analysis (PCA) of the first three principal components in 44 tissues; viz., 11 brains (open circles), 11 kidneys (closed circles), 11 livers (orange circles) and 11 testes (purple circles). The PCA scores of the first principal component (PC1) versus those of the third principal component (PC3) (A), and those of the second principal component (PC2) versus PC3 scores (B) in mouse tissues were plotted. Portrayals of PC1 (blue line), PC2 (red line) and PC3 (green line) loadings indicated their respective peaks with high-low variations (C).
Table1. Peaks at loading of PC1, PC2 and PC3

\begin{tabular}{lll}
\hline PC1 & PC2 & PC3 \\
\hline $630 \uparrow$ & $630 \uparrow$ & $630 \downarrow$ \\
$680 \uparrow$ & $680 \downarrow$ & $670 \uparrow$ \\
$705 \downarrow$ & $705 \downarrow$ \\
& $740 \downarrow$ & $740 \downarrow$ \\
$760 \downarrow$ & $760 \uparrow$ & $760 \uparrow$ \\
$950 \downarrow$ & $780 \downarrow$ & \\
& $930,940 \uparrow$ & $940 \downarrow$ \\
& $960 \downarrow$ & $960 \uparrow$ \\
\hline
\end{tabular}

explained in terms of spectra. Based on the first three loadings (Fig. 2C) and the peaks (Table 1), the loadings of PC1 (high: ca. 630, $680 \mathrm{~nm}$; low: ca. 705, 760, $950 \mathrm{~nm}$ ), PC2 (high: ca. 630, 760, 930, $940 \mathrm{~nm}$; low: ca. 680, 740, 780, $960 \mathrm{~nm}$ ) and PC3 (high: ca. 670, 760, $960 \mathrm{~nm}$; low: ca. 630, $705,740,940 \mathrm{~nm}$ ) varied from high to low with their respective wavelengths. Several peaks were commonly observed between the three PCA loadings. The peaks near $950 \mathrm{~nm}$ have previously been reported to be related to water $[15$, 26], and are commonly observed in PC1 $(950 \mathrm{~nm}), \mathrm{PC} 2$ $(930,940,960 \mathrm{~nm})$ and PC3 $(940,960 \mathrm{~nm})$. The peaks around $750 \mathrm{~nm}$ have reported elements existing close to a water band, and are accordingly observed in PC1 $(760 \mathrm{~nm})$, PC2 $(740,760 \mathrm{~nm})$ and PC3 $(740,760 \mathrm{~nm})$. These results suggest that each tissue showed specific interaction with water, implying absorption of water. Peaks at $630 \mathrm{~nm}$ and $680 \mathrm{~nm}$ were routinely observed in three PCA loadings. The peak near $630 \mathrm{~nm}$ has previously been assigned to $\mathrm{N}-\mathrm{H}$ stretching mode of amides, whereas that near $680 \mathrm{~nm}$ is assigned to $\mathrm{O}-\mathrm{H}$ stretching mode of alcohols [28]. A negative peak at $780 \mathrm{~nm}$ was observed only in PC2 loadings. A peak near $780 \mathrm{~nm}$ has previously been assigned to N-H stretching mode of amides [15]. These results suggest that each tissue also displayed specific interactions with certain organic functional groups. Taken together, this is consistent with the concept that tissue characterization is measured indirectly by absorbance corresponding to the specific interaction between the tissue constituents and water or certain organic compounds.

Differences in the PCA scores among tissues were also observed. The PC2 scores of brain and testes were high, while those of kidneys and livers were low, probably reflecting that differences in blood absorption existed between two groups. Although a peak at $780 \mathrm{~nm}(\mathrm{~N}-\mathrm{H}$ stretching mode of amides) was consistently observed in the PC2 loadings, such was not the case in PC1 and PC3. Moreover, tissues of the PC3 score plots were also divisible into 2 groups (high group: liver and testes; low group: brain and kidney). Although no specific peaks characteristic of $\mathrm{PC} 1$ and $\mathrm{PC} 2$ were observed in PC3, the presence of peaks around $705 \mathrm{~nm}$ and the absence of peaks around $780 \mathrm{~nm}$ were representative of PC3 loadings (cf. PC2). Although it is often difficult to assign wavelengths to specific molecular absorptions in the NIR and red regions, the specific peaks observed in each principal component may be related to specific features of 
biomolecules (such as proteins, lipids, and sugars) produced in tissues.

The present findings suggest that Vis-NIR spectroscopy has the advantage of affording a non-destructive analysis for tissue discrimination. Furthermore, other advantages of Vis-NIR spectroscopy include prompt analysis, simplicity in sample preparations, and non-requirements of chemical reagents. Therefore, Vis-NIR spectroscopy may be used as a large-scale routine assay for discriminating infected tissues. If Vis-NIR spectroscopy apparatus could be automated, the procedural time of yielding a tissue discriminating method would further be abbreviated, thus contributing to the reduction of risks of pathogen contamination from infected tissues.

\section{REFERENCE}

1. Barnes, R.J., Dhanoa, M.S. and Lister, S.J. 1989. Appl. Spectrosc. 43: 772-777.

2. Ciurczak, E.W. and Drennen, J.K. 2002. Pharmaceutical and Medical Applications of Near-Infrared Applications (Pratical Spectroscopy), Marcel Dekker Inc, New York.

3. De Filippo, M., Sudberry, J.J., Borgia, D., Rovani, C., Chernyschova, N., Salati, F. et al. 2005. Acta Biomed. Ateneo. Parmense. 76: 137-151.

4. Eastlund, T. and Strong, D.M. 2004, pp. 51-131. In: Advances in Tissue Banking, (Philips, G.O. ed.), World Scientific, Singapore.

5. Fishman, J.A. 2001. J. Card. Surg. 16: 363-373.

6. Gavier-Widén, D., Stack, M.J., Baron, T., Balachandran, A. and Simmons, M. 2005. J. Vet. Diagn. Invest. 17: 509-527.

7. Goodman, J.L. 2004. New Engl. J. Med. 351: 819-822.

8. Hill, C.R. and Carpenter, D.A. 1976. Br. J. Radiol. 49: 238243.

9. Jolliffe, I.T. 2002. Principal component analysis, 2nd ed, Springer, New York.

10. B.K. Lavine. 2000, pp. 1-21. In: Encyclopedia of Analytical Chemistry : Applications, Theory and Instrumentation (Meyers, R.A. ed.), John Wiley, New York.

11. Long, E.E., Van Dam, J., Weinstein, S., Jeffrey, B., Desser, T. and Norton, J.A. 2005. Surg. Oncol. 14: 105-113.

12. Miller, J.N. and Miller, J.C. 2000. Statistics and Chemometrics for Analytical Chemistry, 4th ed., Prentice Hall, Harlow, UK.

13. Murray, I. 1993, pp. 285-312. In: Sward Management Handbook (Davies, A., Baker, R.D., Grant, S.A. and Laidlaw, A.S. eds.), British Grassland Society, UK.

14. Ory, P.A. 2003. Baill. Best Pract. Res. Clin. Rheumatol. 17: 495-512.

15. Osborne, B.G. and Fearn, T. 1986, Near-Infrared Spectroscopy in Food Analysis, Longman Scietific \& Technical, UK.

16. Pauli, G. 2005. Cell Tissue Bank 6: 191-200.

17. Prusiner, S.B. 1998. Proc. Natl. Acad. Sci. U.S.A. 95: $13363-$ 13383.

18. Sakudo, A., Suganuma, Y., Kobayashi, T., Onodera, T. and Ikuta, K. 2006. Biochem. Biophys. Res. Commun. 341: 279 284.

19. Sakudo, A., Kuratsune, H., Kobayashi, T., Tajima, S., Watanabe, Y. and Ikuta, K. 2006. Biochem. Biophys. Res. Commun. 345: 1513-1516.

20. Sakudo, A., Tsenkova, R., Onozuka, T., Morita, K., Li, S., Warachit, J., Iwabu, Y., Li, G., Onodera, T. and Ikuta, K. 2005. Microbiol. Immunol. 49: 695-701.

21. Savitzky, A. and Golay, M.J.E. 1964. Anal. Chem. 36: 16271639.

22. Siesler, H.W., Ozaki, Y., Kawata, S. and Heise, H.M. 2002. Near-Infrared Spectroscopy (Principles, Instruments, Applications), Wiley-VCH, Weinheim.

23. Srinivasan, A., Burton, E.C., Kuehnert, M.J., Rupprecht, C., Sutker, W.L., Ksiazek, T.G. Paddock, C.D., Guarner, J., Shieh, W.J., Goldsmith, C., Hanlon, C.A., Zoretic, J., Fischbach, B., Niezgoda, M., El-Feky, W.H., Orciari, L., Sanchez, E.Q., Likos, A., Klintmalm, G.B., Cardo, D., LeDuc, J., Chamberland, M.E., Jernigan, D.B., Zaki, S.R. and Rabies in Transplant Recipients Investigation Team. 2005. New Engl. J. Med. 352: 1103-1111.

24. Steinier, J., Termonia, Y. and Deltour, J. 1972. Anal. Chem. 44: 1906-1909.

25. Taylor, L.H., Latham, S.M. and Woolhouse, M.E. 2001. Philos. Trans. R. Soc. Lond. Biol. 356: 983-989.

26. Wheeler, O.H. 1959. Chem. Rev. 59: 629-666.

27. Wold, S. 1976. Pattern Recognit. 8: 127-139.

28. Workman, J. 2001, Handbook of Organic Compounds: NIR, IR, Raman, and UV-Vis Spectra Featuring Polymers and Surfactants, Academic Press, San Diego. 